\title{
THE AXIOM OF DETERMINATENESS AND REDUCTION PRINCIPLES IN THE ANALYTICAL HIERARCHY
}

\author{
BY DONALD A. MARTIN
}

\author{
Communicated by Dana S. Scott, January 12, 1968
}

Let $R$ be the set of all sets of natural numbers. A collection $a$ of subsets of $R$ satisfies a reduction principle if, for every $A$ and $B \in Q$, there are $A^{\prime}$ and $B^{\prime} \in Q$ such that $A^{\prime} \subseteq A, B^{\prime} \subseteq B, A^{\prime} \cup B^{\prime}=A \cup B$, and $A^{\prime} \cap B^{\prime}$ is empty. For $n>0$ let $\Pi_{n}^{1}$ and $\Sigma_{n}^{1}$ be, respectively the set of $\Pi_{n}^{1}$ subsets of $R$ and the set of $\Sigma_{n}^{1}$ subsets of $R$. It is known that $\Pi_{1}^{1}$ and $\Sigma_{2}^{1}$ satisfy reduction principles and that for no $n$ do both $\Pi_{n}^{1}$ and $\Sigma_{n}^{1}$ satisfy reduction principles. (For basic definitions and facts concerning the analytical hierarchy and the degrees of unsolvability, see [5].) Using the Axiom of Constructibility, Addison [1] shows that, for all $n \geqq 2, \Sigma_{n}^{1}$ satisfies a reduction principle. J. Silver has shown that Addison's result is consistent with the assertion that a measurable cardinal exists.

For each $n>0$, let $\Gamma_{n}^{1}$ be $\Pi_{n}^{1}$ if $n$ is odd and $\Sigma_{n}^{1}$ if $n$ is even. For a statement of the Mycielski-Steinhaus Axiom of Determinateness (AD) and proofs of some of its consequences, see [4]. We assume AD and the Axiom of Dependent Choice (DC) and outline a proof that, for every $n, \Gamma_{n}^{1}$ (and hence $\Gamma_{n}^{1}$ ) satisfies a reduction principle. This result has been proved independently by Moschovakis and Addison [2].

Since AD is false, a word is in order about the significance of our proof. In the notation of [4], AD says that, for every $P \subseteq 2^{\omega}, G_{2}(P)$ is determined. Although this contradicts the Axiom of Choice, it remains possible that a very large class of $G_{2}(P)$ are determined. For instance, it is possible that $G_{2}(P)$ is determined for every projective $P$, and this is enough to deduce our result. Indeed, to prove reduction for $\Gamma_{n}^{1}$ we need only assume that $G_{2}(P)$ is determined for every $\Delta_{n-1}^{1} P$. We need $\mathrm{DC}$ for $n \geqq 4$. While $\mathrm{AD}$ may well be consistent with $\mathrm{DC}$, our justification for using $D C$ is rather that we are assuming only a part of $\mathrm{AD}$ which we hope to be consistent with the Axiom of Choice.

Our tool in studying the analytical hierarchy is the Lemma below. Our first proof of reduction for $\Pi_{3}^{1}$ was based on a new proof by Blackwell [3] using infinite games-of reduction for $\Pi_{1}^{1}$. (The methods of [2] are closely related to those of Blackwell.) However, the Lemma provides a different proof which generalizes easily to all odd levels of the hierarchy. The Lemma is a consequence of $\mathrm{AD}$ and is an interesting proposition in its own right. Also, the problem of proving the Lemma consistent (say, assuming large cardinals of some kind) might 
be much easier than the consistency of full AD. (The Lemma is inconsistent with the Axiom of Choice.)

Lemma. Let $\mathcal{E} \subseteq \mathbb{D}$, the set of all degrees of unsolvability. There is a degree $d_{0}$ such that either $d \geqq d_{0} \rightarrow d \in \mathcal{E}$ or $d \geqq d_{0} \rightarrow d \in D-\mathcal{D}$.

Proof (Assuming AD). Let $P$ be the set of all sequences $\in 2^{\omega}$ whose degree of unsolvability belongs to $\varepsilon$. We consider the game $G_{2}(P)$. Suppose for definiteness that I has a winning strategy. The strategy is essentially a number-theoretic function. Let $\boldsymbol{d}_{0}$ be its degree of unsolvability. We show that $d \geqq d_{0} \rightarrow d \in \mathcal{E}$. Let $d \geqq d_{0}$ and let $\alpha$ be a sequence of degree $d$. Suppose II plays $\alpha$ and I plays according to his strategy. The sequence produced has degree $d$. Hence $d \in \mathcal{E}$.

The Lemma yields a countably additive zero-one measure, which we call $\mu$, on the degrees. $\mu(\varepsilon)=1$ provided that every sufficiently large degree belongs to $\varepsilon$. (The particular measure $\mu$ is very important in the sequel. Many other measures on $D$ can be defined using AD.) To prove a reduction principle for each $\Gamma_{n}^{1}$, we simply use $\mu$ to continue past level 2 the familiar process of assigning ordinal numbers to $\Gamma_{n}^{1}$ sentences. This assignment of ordinals is enough not only to prove reduction principles but also to lift much of the theory of $\Pi_{1}^{1}$ and $\Sigma_{2}^{1}$ up to a theory of $\Gamma_{n}^{1}$. (See [2] for details.) The delicate theorems about $\Pi_{1}^{1}$, such as the Kondo-Addison Theorem, seem, however, to require more than just this assignment of ordinals.

For each $n>0$ and $m \geqq 0$, let $W_{n}^{m}$ be the set of all $\left\langle e, \alpha_{1}, \cdots, \alpha_{m}\right\rangle$ such that $e$ is the Gödel number of a $\Gamma_{n}^{1}$ formula $A$ of $m$ set variables and $A\left(\alpha_{1}, \cdots, \alpha_{m}\right)$ holds. We sometimes write $\left\langle e, \alpha_{1}, \cdots, \alpha_{m}\right\rangle$ as $\langle e, \boldsymbol{\alpha}\rangle$ or $A(\alpha)$. By induction on $n$, we assign ordinals $|e, \alpha|^{n}$ to the members $\langle e, \alpha\rangle$ of $W_{n}^{m}$. The assignment of ordinals will have two properties:

(1) The relations $\left|e_{1}, \alpha_{1}\right|^{n}<\left|e_{2}, \alpha_{2}\right|^{n}$ and $\left|e_{1}, \alpha_{1}\right|^{n} \leqq\left|e_{2}, \alpha_{2}\right|^{n}$ are $\Gamma_{n}^{1}$.

(2) For $\left\langle e_{2}, \alpha_{2}\right\rangle \in W_{n}^{m}$, the complements of $\left|e_{1}, \alpha_{1}\right|^{n}<\left|e_{2}, \alpha_{2}\right|^{n}$ and $\left|e_{1}, \boldsymbol{\alpha}_{1}\right|^{n} \leqq\left|e_{2}, \boldsymbol{\alpha}_{2}\right|^{n}$ are $\Gamma_{n}^{1}$ uniformly in $\left\langle e_{2}, \boldsymbol{\alpha}_{2}\right\rangle$.

For $n=1$, the assignment of ordinals is a standard procedure. There is also a well-known method for using the assignment for $\Pi_{1}^{1}$ to get an assignment for $\Sigma_{2}^{1}$. This method is perfectly general and allows us to go from $\Pi_{n}^{1}$ to $\Sigma_{n+1}^{1}$ : let $|(\exists \alpha) A(\alpha, \alpha)|^{n+1}=\inf \left\{|A(\alpha, \alpha)|^{n}: A(\alpha, \alpha)\right\}$. We omit the details.

Let $n \geqq 3$ be odd. Let $(\alpha) A(\alpha, \alpha) \in W_{n}^{m}$. For each $\alpha \in R$, let $d(\alpha)$ be the degree of $\alpha$. We assign to each degree of unsolvability $d$ an ordinal $|A(\boldsymbol{d}, \boldsymbol{\alpha})|^{n-1}$ as follows:

$$
|A(d, \alpha)|^{n-1}=\sup \left\{|A(\alpha, \alpha)|^{n-1}+1: d(\alpha) \leqq d\right\} .
$$


By induction, the predicate

$$
|B(d(\beta), \beta)|^{n-1}<|A(d(\alpha), \alpha)|^{n-1}
$$

is easily seen to be $\Delta_{n-1}^{1}$ uniformly in $\langle A, \alpha, \alpha\rangle$; similarly for $\leqq$. We define $|A(\alpha)|^{n}$ by stipulating that

$$
\begin{aligned}
|(\beta) B(\beta, \beta)|^{n} & <|(\alpha) A(\alpha, \alpha)|^{n} \\
\leftrightarrow & \mu\left(\left\{d:|B(d, \beta)|^{n-1}<|A(d, \alpha)|^{n-1}\right\}\right)=1 \\
\leftrightarrow & (\alpha) A(\alpha, \alpha) \&\left(\alpha^{\prime}\right)(\exists \alpha)\left(d\left(\alpha^{\prime}\right)\right. \\
& \left.\leqq d(\alpha) \&|B(d(\alpha), \beta)|^{n-1}<|A(d(\alpha), \alpha)|^{n-1}\right) \\
\leftrightarrow & (\alpha) A(\alpha, \alpha) \&\left(\exists \alpha^{\prime}\right)(\alpha)\left(d\left(\alpha^{\prime}\right) \leqq d(\alpha)\right. \\
& \left.\rightarrow|B(d(\alpha), \beta)|^{n-1}<|A(d(\alpha), \alpha)|^{n-1}\right) .
\end{aligned}
$$

If $<$ is well founded, we can simply assign the least ordinals consistent with $<$. But the well-foundedness of $<$ is just a standard fact about ultraproducts with respect to countably additive measures. If $<$ were not well-founded, by DC there would be a sequence $\left|(\alpha) A_{1}\left(\alpha, \alpha_{1}\right)\right|^{n}>\left|(\alpha) A_{2}\left(\alpha, \alpha_{2}\right)\right|^{n}>\cdots$. Hence, on a set of measure one, $\left|A_{1}\left(d, \alpha_{1}\right)\right|^{n-1}>\left|A_{2}\left(d, \alpha_{2}\right)\right|^{n-1}>\cdots$.

Reduction for $\Gamma_{n}^{1}$ is now easy. Let $A=\{\alpha: A(\alpha)\}$ and $B=\{\alpha: B(\alpha)\}$, where $A(\alpha)$ and $B(\alpha)$ are $\Gamma_{n}^{1}$. Let $A^{\prime}=\left\{\alpha: A(\alpha) \& 7|B(\alpha)|^{n}<|A(\alpha)|^{n}\right\}$ let $B^{\prime}=\left\{\alpha: B(\alpha) \& 7|A(\alpha)|^{n} \leqq|B(\alpha)|^{n}\right\}$.

We do not know whether AD $+D C$ implies uniformization for $\Gamma_{n}^{1}$. We conjecture that it does. $R$. Solovay and the author have shown that $A D+D C$ does yield one new basis theorem: Every nonempty $\Sigma_{3}^{1}$ set has a $\Delta_{4}^{1}$ member. However, our proof does not really need AD, but requires only that a Ramsey cardinal exists.

\section{REFERENCES}

1. J. W. Addison, Separation principles in the hierarchies of classical and effective descriptive set theory, Fund. Math. 46 (1958), 123-135.

2. J. W. Addison and Y. N. Moschovakis, Some consequences of the axiom of definable determinateness, Proc. Nat. Acad. Sci. U.S.A. 59 (1968), 708-712.

3. D. Blackwell, Infinite games and analytic sets, Proc. Nat. Acad. Sci. U.S.A. 58 (1967), 1836-1837.

4. J. Mycielski, On the axiom of determinateness, Fund. Math. 53 (1964), 205-224.

5. H. Rogers, Jr., Theory of recursive functions and effective computability, McGrawHill, New York, 1967.

ROCKEFELLER UNIVERSITY 